\title{
Multi-modality assessment of mechanical complications in a patient with inferior myocardial infarction - Case Report
}

Nilda Espinola-Zavaleta, Joaquin Berarducci*, Jose Antonio Luna-Alvarez-Amézquita, Javier Ivan Armenta-Moreno, Mara EscuderoSalamanca and Jose Luis Siller-Nava

Department of Nuclear Cardiology, National Institute of Cardiology Ignacio Chávez, Mexico City, Mexico

\begin{abstract}
A postmenopausal female patient with a non-treated STEMI that developed mechanical complications (ventricular septal rupture and left ventricular aneurysm) with acute onset heart failure. The diagnosis was made because of her overload symptoms (dyspnea, distal edema) 1 month after her STEMI. Electrocardiogram, TTE, MRI and angiography were made with concluding results. The patient was treated with diuretics and a percutaneous transcatheter ventricular septal occluder device with successful hemodynamic and clinical outcomes. The percutaneous device placement was decided due to the severity of the symptoms and the association of her mechanical complications.
\end{abstract}

Abbreviations: STEMI: ST-elevation myocardial infarction; TTE: Transthoracic echocardiogram; MRI: Magnetic resonance imaging; ECG: Electrocardiogram; CTA: Computed tomography angiography; VSR: Ventricular septal rupture; NYHA: New York Heart Association.

\section{Introduction}

Acute myocardial infarction (MI) is the leading cause of dead in the world, but the mechanisms which produces the morbidity and mortality associated with MI are not always the same. The incidence of complications posterior to an MI increase when the treatment of the event is significantly delayed or inadequate. In our era, where percutaneous coronary intervention is the treatment of choice for the management of acute ST elevation myocardial infarctions, the secondary complications have been reduced to less than $1 \%$ [1].

The most important complications can be mainly divided into electrical, mechanical, inflammatory, and embolic categories. At the same time, the complications we should bring to our minds posterior to MI depend on the time elapsed following the event. ${ }^{2}$ In the acute presentation of the disease, the most common complications secondary to the myocardial insult are the arrythmias, in which ventricular tachycardia and ventricular fibrillation account for most cases of sudden cardiac death following a myocardial infarction [2].

In mechanical complications of MI, the timing of the clinical presentation is key for establishing the differential diagnosis and deciding the urgency, type of treatment, and imaging required for the management of patients. In the acute phase, the most frequent complications are ventricular free wall rupture, ventricular septal defect, and papillary muscle rupture [3]. In the other hand, when temporality favors a chronic course, the associated complications are left ventricular aneurysm, pseudoaneurysms, and mitral regurgitation. The importance of acknowledging the existence of this complications despite their low frequency is due to the implications on prognosis for patients suffering from them, and to offer treatment options at the proper time [4].

\section{Case Report}

\section{Patient presentation}

50-year-old postmenopausal female patient with history of type 2 diabetes mellitus treated with glargine 22 units daily and hypertension of 7 years evolution, without treatment. She was admitted in a local hospital because she had chest pain, associated with exertional shortness of breath, radiated to left arm. On the electrocardiogram (ECG) an inferior ST elevation myocardial infarction was detected. She received medical treatment with Aspirin $100 \mathrm{mg}$ daily, spironolactone $25 \mathrm{mg}$ daily, isosorbide dinitrate $10 \mathrm{mg}$ every 12 hours, Atorvastatin $20 \mathrm{mg}$ daily, Clopidogrel $75 \mathrm{mg}$ daily and furosemide $20 \mathrm{mg}$ daily, no re-perfusive treatment was given. After two weeks of the myocardial infarction, she started with exertional and paroxysmal nocturnal dyspnea, progressive distal edema, and palpitations. She was admitted in the emergency care unit again. The physical examination revealed blood pressure of 140/90 $\mathrm{mmHg}$, heart rate 115 beats/min, respiratory 20 breaths/min, temperature $36.6^{\circ} \mathrm{C}$, oxygen saturation $92 \%$ on room air, jugular engorgement, fine crackles in both lung bases and a holosystolic murmur III/IV in all mesocardium not heard in her previous visit and severe bilateral distal edema up to the knee level. She was diagnosed with acute heart failure with bilateral pleural effusion. Furosemide dose was given $(40 \mathrm{mg})$ and a bilateral thoracentesis was performed draining $1100 \mathrm{ml}$ on the right side and $600 \mathrm{ml}$ on the left side.

*Correspondence to: Joaquin Berarducci MD, Department of Nuclear Cardiology, National Institute of Cardiology Ignacio Chávez, Mexico City, Mexico, E-mail: jberarducci96@gmail.com

Key words: STEMI, ventricular septal rupture, ventricular aneurysm, myocardial infarction, acute heart failure

Received: June 08, 2021; Accepted: June 17, 2021; Published: June 21, 2021 


\section{Initial work up}

ECG with sinus rhythm, heart rate $80 \mathrm{bpm}$, Q waves in DII, DIII and AVF with an interventricular conduction delay and subepicardial ischemia in the inferior wall (Figure 1). Laboratory tests showed hemoglobin of 13.4 grams/dL, hematocrit $41.7 \%$, leukocytes 7.1 $\mathrm{x} 10^{9} / \mathrm{L}$, platelets 203,000 per microliter, creatinine $0.77 \mathrm{mg} / \mathrm{dL}$, calcium $8.15 \mathrm{mg} / \mathrm{dL}$, potassium $3.27 \mathrm{mg} / \mathrm{dL}$, sodium $140 \mathrm{mg} / \mathrm{dL}$ glucose $159 \mathrm{mg} / \mathrm{dL}$, BUN $26.81 \mathrm{mmol} / \mathrm{L}$, and troponin I $31.4 \mathrm{ng} / \mathrm{mL}$. Chest $\mathrm{X}$ ray showed bilateral pleural effusion with mild cardiomegaly. Transthoracic echocardiogram (TTE) revealed a left ventricular inferobasal aneurysm, postero-lateral VSR with left to right shunt and Qp/ Qs of 3.2, infero-lateral hypokinesia and left ventricular systolic dysfunction. Computed tomography angiography (CTA) showed coronary calcium agatston score of 69.3, an aneurysm of the basal and mid thirds of the infero-basal wall with significant thinning of the wall and muscular inferior VSR. Angiography demonstrated dominant left circumflex artery with a calcified total occlusion with antegrade flux in its distal segment. Multiple lesions in the entire artery with TIMI 2. Ventriculography evidenced VSR and left ventricular aneurysm.

\section{Diagnosis and Management}

The patient was diagnosed with a Q- wave myocardial infraction in the inferior wall that was not treated acutely due to delayed arrival to the emergency room, therefore, mechanical complications occurred. The TTE showed an interventricular septal rupture with an infero-basal aneurysm. Angiography and CTA showed a total distal occlusion of the left circumflex artery (Figure 2). The patient remained with dyspnea, distal edema and pleural effusion, and there was enough evidence of the mechanical dysfunction. The acute onset right heart failure that caused the hemodynamic and clinical overload changes was initially treated with intravenous diuretics as the guidelines suggest $(20$ mg of Furosemide every 12 hours) without hypotensive episodes [5]. A percutaneous approach was decided due to its lower risk of mortality and the patient's condition without any contraindications [6]. A transcatheter VSR device closure was successfully done, the residual Qp/Qs was 1.2. A pleural catheter was placed to treat the right pleural effusion. Enoxaparin $40 \mathrm{mg}$ was given daily during her hospitalization.

\section{Follow-up}

After the procedure the patient remained asymptomatic with no further distal edema neither dyspnea of any kind, and with adequate urinary output with $10 \mathrm{mg}$ of furosemide every 12 hours given to improve her bilateral pleural effusion. Transthoracic echocardiogram and cardiac magnetic resonance (Figures 3 and 4) showed VSR occluder device placed in the interventricular septum without evidence of residual shunt, there was no evidence of thrombi within the aneurysm, moderate tricuspid regurgitation and left and right ventricular systolic dysfunction (LVEF-42\%, RVEF-37\%), Video 1. The pleural effusion improved so the pleural catheter was taken off. She had great outcomes with expected results and clinical improvement and had outpatient follow up with the following treatment: salicylic acid $100 \mathrm{mg}$ daily, clopidogrel $75 \mathrm{mg}$ daily, atorvastatin $80 \mathrm{mg}$ daily. In the fifteen-month follow up the patient remains asymptomatic.

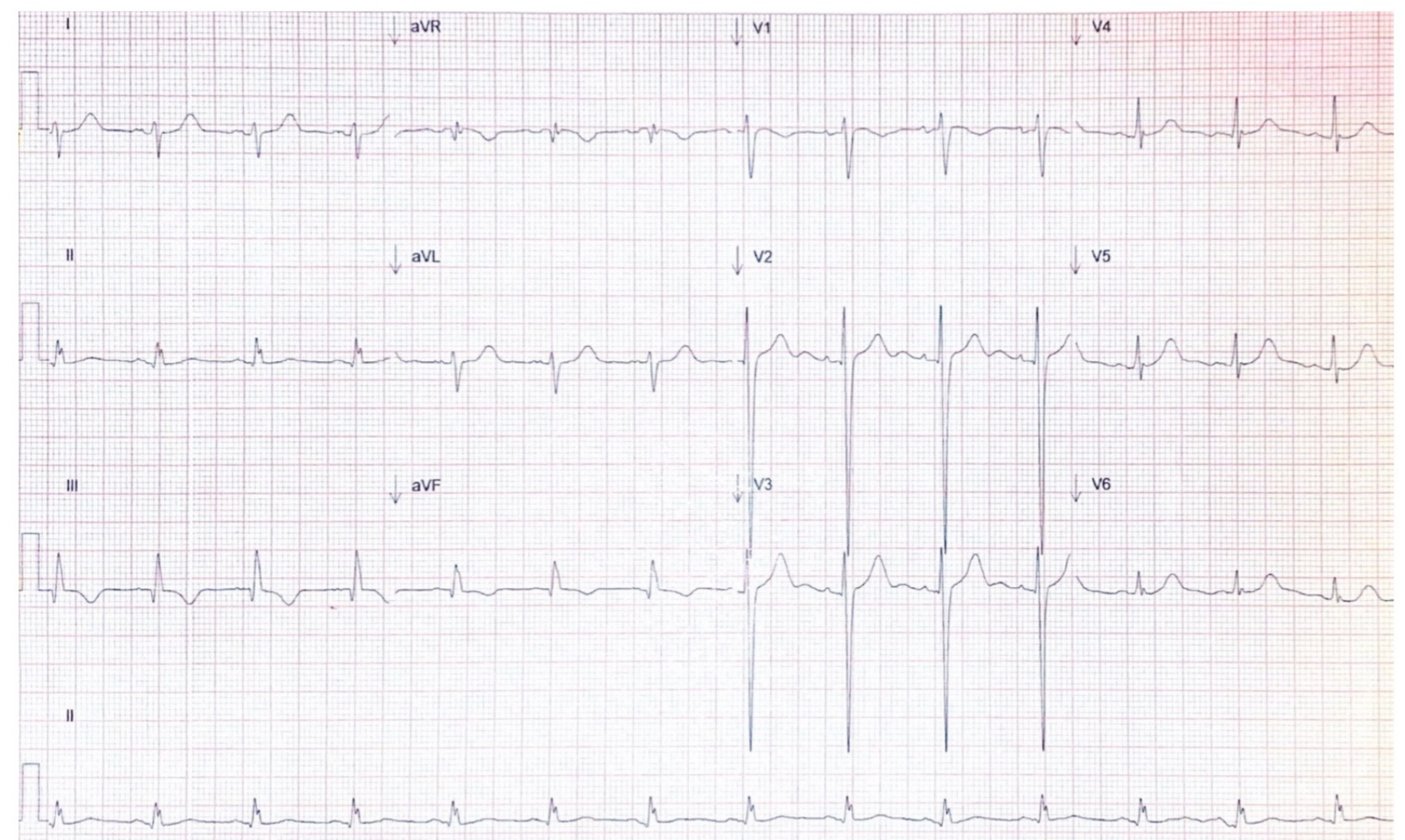

Figure 1. Electrocardiogram. ECG with sinus rhythm, heart rate $80 \mathrm{bpm}$, Q waves in DII, DIII and aVF, an interventricular conduction delay and subepicardial ischemia in the inferior wall 


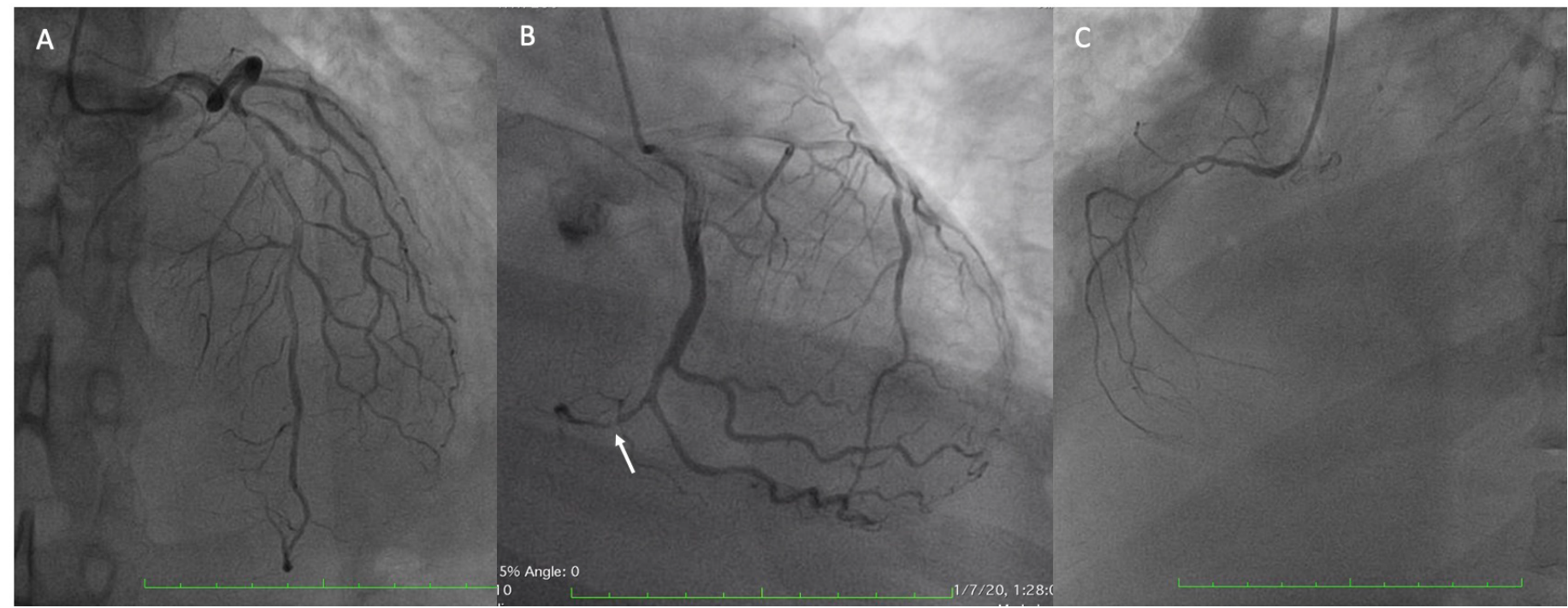

Figure 2. Coronary angiography showing: A) left anterior descending artery without significant lesions, B) dominant circumflex artery with disease along its entire length and with anterograde recanalized distal total occlusion (arrow), C) Right coronary artery without significant lesions

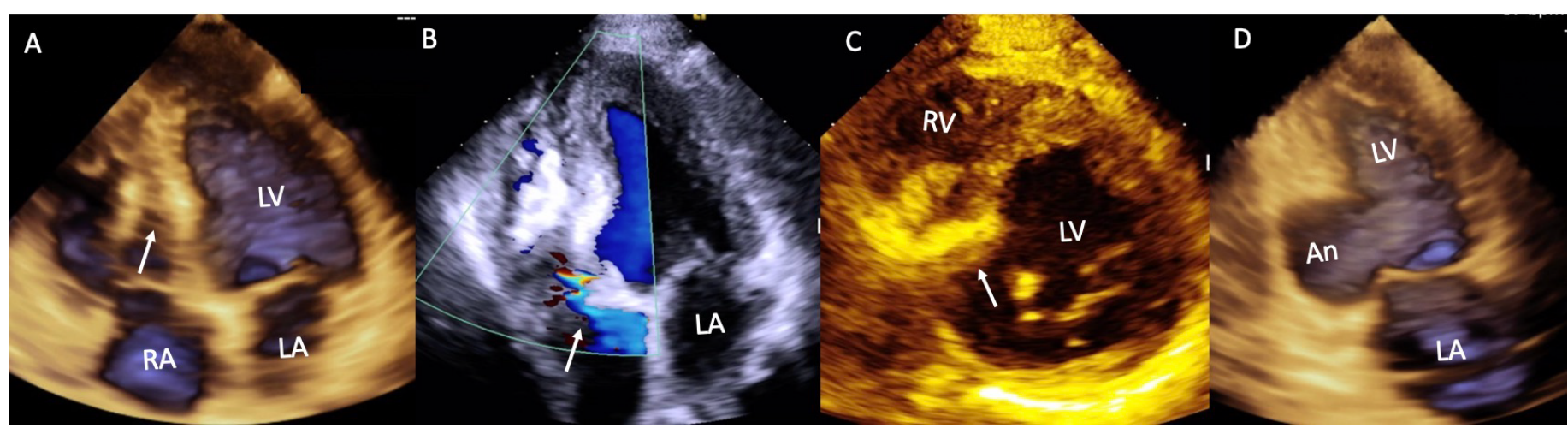

Figure 3. Bidimensional and three-dimensional transthoracic echocardiogram. A) 3D-four chamber view with occluder device placed in the interventricular septum (arrow), B) with color flow without residual shunt and moderate tricuspid regurgitation (arrow), C) short axis view showing the occluder device placed in the basal portion of the interventricular septum, D) 3D-two-chamber view with an aneurysm in the basal portion of the left ventricular inferior wall

Abbreviations: RA-right atrium, RV-right ventricle, LA-left atrium, LV-left ventricle, An-aneurysm

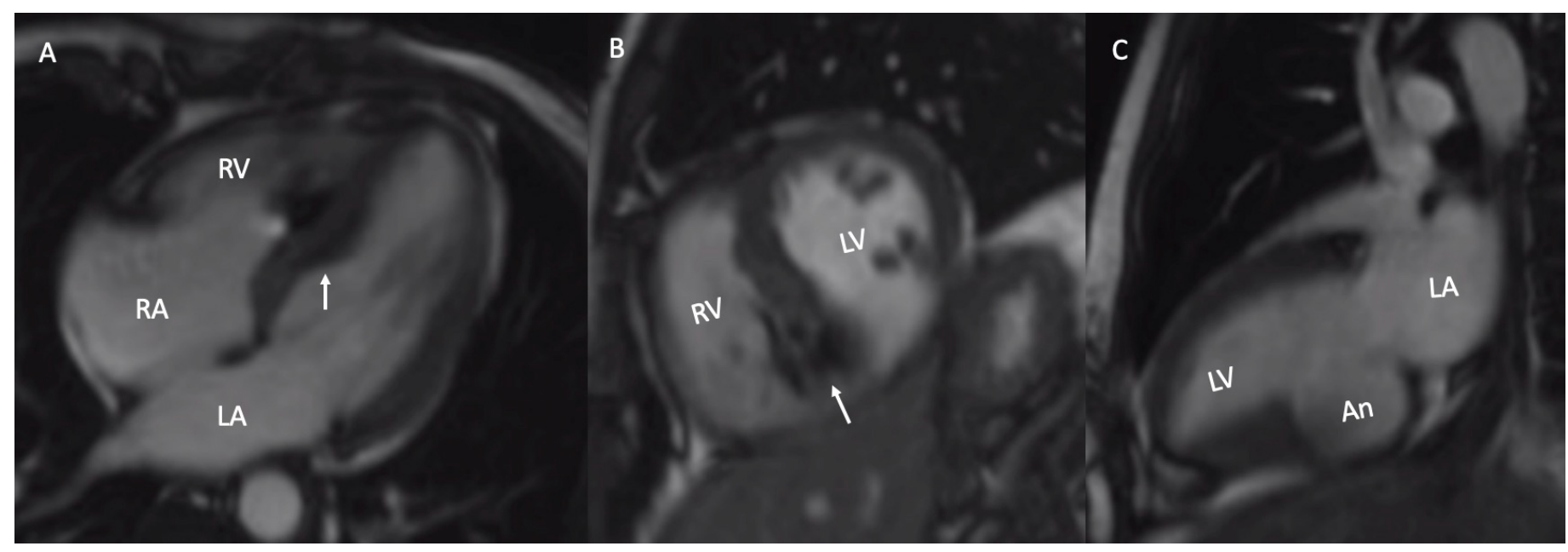

Figure 4. Magnetic resonance imaging. A) four-chamber view B) short-axis view at the level of both ventricles showing the occluder device placed in the basal portion of the interventricular septum, C) two-chamber view with a left ventricular infero-basal aneurysm

Abbreviations: RA-right atrium, RV-right ventricle, LA-left atrium, LV-left ventricle, An-aneurysm 


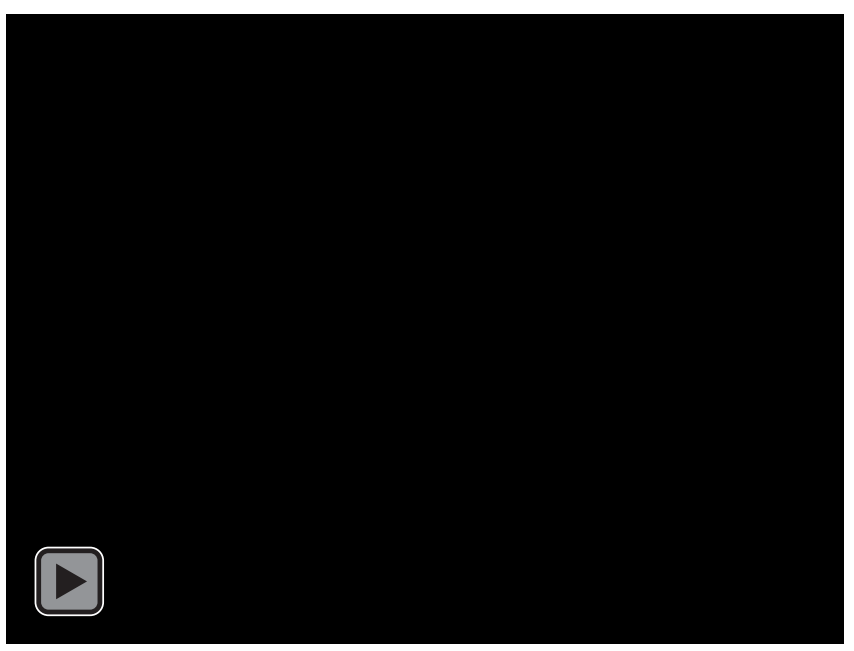

Video 1. TTE three-dimensional four chamber view showing the correct implantation of the occluding device in the interventricular septum

\section{Discussion}

We present the case of a female patient with two mechanical complications after a myocardial infarction. It was previously estimated that left ventricular aneurysms developed in $35 \%$ of patients with Q-wave myocardial infarction [7], however, the incidence of this complication is decreasing and currently is about $10 \%$ for these patients [8]. Our patient developed the most common type of left ventricular aneurysm involving the inferior wall. This was illustrated in a review of 52 patients seen at the Mayo Clinic. Aneurysms were primarily seen in the inferior or postero-lateral wall after MI, which is consistent with inferior infarction, in the right ventricular outflow tract after congenital heart surgery, in the posterior sub-annular region of the mitral valve after mitral valve replacement, and in the subaortic region after aortic valve replacement [9].

Mechanical complications occur at a rate of about 3 per 1000 patients with acute MI, and most of these events are detected in patients with STEMI. Among these patients, ventricular septal rupture is the most common and occurs with a frequency of about 1 in 1000 , free wall rupture is the least common [10]. An intracardiac shunt is hemodynamically significant if: there is evidence of cavity enlargement, and/or evidence of sustained Qp:Qs $\geq 1.5: 1$. An intracardiac shunt not meeting these criteria would be described as small or trivial. Since our patient presented de aforementioned criteria, the defect was closed percutaneously [11].

TTE is often the first test of choice in a patient with suspected mechanical complications after a MI, since it's a non-invasive technique that can be performed at bedside. In the case of VSR it is sufficient to identify the presence, region, and size of the rupture, direction of the shunt, cavity enlargement, and pulmonary hypertension. For ventricular aneurysms TTE is also the first imaging modality used. The difference between pseudoaneurysms and true aneurysms is the size of the neck, the latter has a wider neck. If there is no clear differentiation further assessment with TEE, CTA or MRI should be performed.

ST elevation myocardial infarctions (STEMI) must be treated as soon as possible to avoid mechanical and functional complications. Acute heart failure symptoms can suggest any of these complications especially in a non-re-perfused patient. A complete diagnostic approach can determine the source of acute onset symptoms and lead to immediate treatment.

\section{Conclusion}

Our patient did not receive re-perfusive therapy when the STEMI occurred. She developed mechanical complications (ventricular aneurysm and ventricular septal rupture) that were treated optimally with a percutaneous transcatheter device. The choice between surgical or percutaneous approach should be made by the patient's individual conditions and medical expertise. Percutaneous device placement was the chosen therapy due to the patient clinical status. The follow- up studies showed hemodynamic improvement with no residual shunt, demonstrating that the interventional treatment was successful.

\section{Funding}

None.

\section{Disclosures}

None.

\section{Conflict of Interest}

None.

\section{Acknowledgments}

None.

\section{References}

1. Stephens NR, Restrepo CS, Saboo SS, Baxi AJ (2019) Overview of complications of acute and chronic myocardial infarctions: revisiting pathogenesis and cross-sectional imaging. Postgraduate Medical Journal, BMJ Journals 2018: 136279.

2. Montrief T, Davis WT, Koyfman A, Long B (2019) Mechanical, inflammatory, and embolic complications of myocardial infarction: An emergency medicine review. Am J Emerg Med 37: 1175-1183. [Crossref]

3. Elbadawi A, Elgendy IY, Mahmoud K, Barakat AF, Mentias A, et al. (2019) Temporal Trends and Outcomes of Mechanical Complications in Patients With Acute Myocardial Infarction. JACC Cardiovasc Interv 12:1825-1836. [Crossref]

4. Al-Naabi MJ, Maddali MM, Venkatachlam R (2021) Mechanical Complication After a Myocardial Infarction. J Cardiothorac Vasc Anesth 35: 670-672. [Crossref]

5. Esc Study Group (2017) The Task Force for The Management of Acute Myocardial Infarction in Patients Presenting with St-Segment Elevation Of The European Society Of Cardiology (Esc) 2017 Esc Guidelines For The management Of Acute myocardial Infarction In Patients Presenting With St-Segment Elevation. Russian Journal of Cardiology 5: 103-58.

6. Daxin Z, Pan W, Guan L, Pan C, Ge J (2015) Transcatheter Closure of a PostMyocardial Infarction Ventricular Septal Rupture Using a Parachute Device. JACC Cardiovasc Interv 2: 369-371. [Crossref]

7. Mills NL, Everson CT, Hockmuth DR (1993) Technical advances in the treatment of left ventricular aneurysm. Ann Thorac Surg 55: 792-800. [Crossref]

8. Myoung M (2019) Left Ventricular Aneurysm Caused by Transmural Myocardial Infarction. Radiologic technology 90: 417-420.

9. Yeo TC, Malouf JF, Oh JK, Seward JB (1998) Clinical Profile and Outcome in 52 Patients with Cardiac Pseudoaneurysm. Ann Intern Med 4: 299-305. [Crossref]

10. Formica, Francesco, Serena Mariani, and Stefano D'Alessandro (2019) Acute Mechanical Complications in Patients Suffering from Acute Myocardial Infarction. Vessel Plus 3: 37.

11. Stout KK, Daniels CJ, Aboulhosn JA, Bozkurt B, Broberg CS, et al. (2018) 2018 AHA ACC Guideline for the Management of Adults with Congenital Heart Disease: A Report of the American College of Cardiology/American Heart Association Task Force on Clinical Practice Guidelines. Circulation 139: 14.

Copyright: (C2021 Espinola-Zavaleta N. This is an open-access article distributed under the terms of the Creative Commons Attribution License, which permits unrestricted use, distribution, and reproduction in any medium, provided the original author and source are credited. 